\title{
Elastic interaction between twins during tensile deformation of austenitic stainless steel
}

Nicolai Ytterdal Juul ${ }^{a}$, Grethe Winther ${ }^{a}$, Darren Dale ${ }^{b}$, Margaret K. A. Koker ${ }^{b}$, Paul Shade ${ }^{c}$ and Jette Oddershede $\mathrm{d}^{\mathrm{d}, *}$

${ }^{a}$ Department of Mechanical Engineering, Technical University of Denmark, Produktionstorvet, 2800 Kgs. Lyngby, Denmark

${ }^{\mathrm{b}}$ Cornell High Energy Synchrotron Source, Ithaca, NY 14853, USA

${ }^{\mathrm{c}}$ Materials and Manufacturing Directorate, Air Force Research Laboratory, Wright-Patterson AFB, OH 45433, USA

${ }^{d}$ Department of Physics, Technical University of Denmark, Fysikvej, 2800 Kgs. Lyngby, Denmark

*Corresponding author. Tel.: +45 2371 2331; fax: +45 4593 2399; email: jeto@fysik.dtu.dk

\begin{abstract}
In austenite, the twin boundary normal is a common elastically stiff direction shared by the two twins, which may induce special interactions. By means of three-dimensional $X$-ray diffraction this elastic interaction has been analysed and compared to grains separated by conventional grain boundaries. However, the components of the Type II stress normal to the twin boundary plane exhibit the same large variations as for the grain boundaries. Elastic grain interactions are therefore complex and must involve the entire set of neighbouring grains. The elastic-regime stress along the tensile direction qualitatively depends on the grain orientation, but grain-to-grain variations are large.
\end{abstract}

\section{Keywords}

Tension test; three-dimensional X-ray diffraction (3DXRD); austenitic steels; elastic behaviour; twinning 
The method of three-dimensional X-ray diffraction (3DXRD) is capable of producing large maps of grain structures [1-3], including crystallographic orientations and neighbour relations [4,5], and also allows measurement of the elastic strain tensor at the level of each individual grain during deformation [6-9]. The average stress tensor of each grain, known as the Type II stress tensor, may subsequently be derived. This gives a unique possibility of investigating elastic grain interactions. Such studies are motivated by line broadening studies, which reveal substantial deviations from the predictions of current elastic grain interaction models based on the orientations of the grains with respect to the tensile axis $[10,11]$.

The present study attempts to study these complex elastic interactions by focusing on the interaction across annealing twin boundaries produced by recrystallization in $316 \mathrm{~L}$ austenitic steel during uniaxial tension. Twin boundaries in the fcc crystal lattice are characterised by a well-defined crystallographic boundary plane, with a normal which happens to be the elastically stiffest $<111\rangle$ direction. The crystallographic misorientation between twins is a $60^{\circ}$ rotation around the twin boundary normal, enabling selection of twin pairs for this study in which one part of the twin pair has its tensile axis aligned close to the elastically most compliant crystallographic $<100>$ direction while the direction of the tensile axis of the other part is significantly stiffer. As a direct consequence of the crystallography of this selection, the inclination of the twinning plane to the tensile axis of the experiment is restricted to a range of $45-65^{\circ}$, ensuring not only crystallographically similar boundary planes, but also boundary planes that are comparable with respect to the macroscopically applied deformation.

The $316 \mathrm{~L}$ austenitic steel material employed has a mean grain size of $70 \mu \mathrm{m}$. The texture is almost random, which further allows for comparison with the elastic interaction across conventional grain boundaries separating compliant grains with crystallographic tensile axes near $<100>$ from stiffer neighbours, while having the same orientation of the grain boundary plane in the deformation system as the twin boundaries.

The diffraction experiment was performed at the F2 line of the Cornell High Energy Synchrotron Source using the RAMS2 load frame [12] to deform the sample in tension while allowing a full $360^{\circ}$ illumination of the $0.7 \times 0.7 \mathrm{~mm}^{2}$ sample cross section. An X-ray beam of $55.681 \mathrm{keV}$ (Ho K-edge) limited with a rectangular slit to $0.10 \mathrm{~mm}$ height by $2.0 \mathrm{~mm}$ width was used to illuminate five consecutive $0.10 \mathrm{~mm}$ thick vertical slices of the sample. Two detectors with a resolution of $2048 \times 2048$ pixels were used separately: one near-field detector (QImaging Retiga 4000DC with an infinity-corrected 5x objective lens) with an effective pixel size of $1.48 \times 148 \mu \mathrm{m}^{2}$ at a distance of $5.1 \mathrm{~mm}$ and one large-area far-field detector (GE Revolution 41RT [13]) with a pixel size of $200 \times 200 \mu \mathrm{m}^{2}$ at a distance of $700 \mathrm{~mm}$ from the specimen. Both detectors were used to characterize the undeformed state of the sample; the near-field detector for data on grain shapes and positions, and the far-field detector for data on grain orientations, elastic strains and stresses. The sample was then deformed in the elastic-regime in tension to $0.12 \%$ total strain confirmed by digital image correlation [14] and $168 \mathrm{MPa}$ according to the RAMS2 load cell, and a second set of far-field data was collected to determine the stress states of the individual grains within the sample. The diffraction data were reconstructed using the FABLE software package. Grainspotter [15] was used for indexing and FitAllB [6] for fitting of the average elastic strain tensor in each individual grain from the far-field data, after which the Type II stress tensor was derived by using the following values for the stiffness tensor: $C_{11}=206$ GPa, $C_{12}$ $=133 \mathrm{GPa}$, and $\mathrm{C}_{44}=119 \mathrm{GPa}$ [16]. The 3D grain morphologies were reconstructed from the near-field data by employing a 3D generalisation of Grainsweeper [4]. The 3D grain map of the illuminated $0.7 \times 0.7 \times 0.5$ $\mathrm{mm}^{3}$ sample volume with a completeness of $70 \%$ can be seen in Figure 1 (a). 
The tensile elastic strain and Type II stress components of each grain in the mapped volume are given in Figure 2 as a function of the crystallographic direction of the tensile axis. It is seen that neither the strain nor the stress components are constant for all grains, implying that the elastic grain interaction lies in between the grain interaction models of Voigt [17] and Reuss [18] assuming strain compatibility and stress equilibrium, respectively. Grains near $<100>$ clearly tend to have the largest tensile strain and the smallest tensile stress, whereas the trend near $<111>$ is opposite. This is in good agreement with the expectation that the $\langle 100\rangle$ oriented grains are the most compliant and those near $<111\rangle$ the stiffest. However, the scatter in both strain and stress values is substantial. The crystallographic direction of the tensile axis thus has an influence on the deformation behaviour of individual grains, but local neighbourhood interactions have a large impact as well

In order to investigate the interaction between twins, the 3D space filling grain map is analysed to unambiguously identify twinned grains using both the determined boundary plane and the crystallographic misorientation. A representative example of a twin pair is presented in Figure 1(b). The twin planes and normal vector have been illustrated for clarity. Figure 3(a) shows the crystallographic orientations of the tensile axes of the 36 twin pairs selected for this study. The compliant part of the twin pair (near $<100>$ ) is coloured red and the stiffer part is coloured blue or green. For those coloured blue, the ratio between the single crystal elastic moduli along the tensile axes of the two parts of the twin pair exceeds 1.7. The green colour represents twins where this ratio lies between 1.7 and 1.2. For comparison with the interaction across conventional grain boundaries, Figure $3(b)$ presents a set of 24 reference grain boundaries connecting 18 compliant grains (as marked in red) to 22 stiffer grain orientations (marked in grey) without fulfilling the boundary plane and crystallographic misorientation criteria for twinning. These reference grain boundaries are inclined $45-65^{\circ}$ to the direction of applied tensile stress like the twin boundaries of the selected twin pairs. Figure 1 (c) shows a representative example of such a pair of neighbouring grains with the grain boundary plane and normal vector indicated for clarity.

With respect to the stress normal to the twin planes of the 36 selected twin pairs, Figure 4(a) shows this stress component in the stiff part plotted versus that in the compliant part. By analogy with the stress along the tensile axis there is significant scatter in the data. For about half of the twin pairs, the stress values cluster in the range 0-100 $\mathrm{MPa}$. However, in the remaining twin pairs considerably larger stresses are observed in either the compliant or the stiff part. These parts often - but far from always - have a small volume. The weighted correlation coefficients are $r^{2}=0.03$ for the 20 twin pairs with largest difference in elastic moduli (blue) and $r^{2}=0.00005$ for the remaining 16 twin pairs (green). Repeating the analysis with only the data clustering in the range 0-100 MPa still gives a vanishing correlation coefficient. This means that the normal stresses across twin boundaries exhibit large variations and that a systematic trend cannot be discerned.

For comparison, Figure 4(b) shows the normal stress across the 24 selected grain boundary planes, with the normal stress in the stiffer grain (grey in Figure 3(b)) plotted against the normal stress in the compliant grain (red in Figure 3(b)). Qualitatively, this figure appears comparable to the same plot for the twins. This is confirmed by the weighted correlation coefficient of $r^{2}=0.002$. Although the mean normal stress in both the compliant and stiff parts are smaller for the twin pairs than for the grain boundaries, as is also the case for the tensile stress components, the spread is too large to make this difference statistically significant. 
The analysis thus concludes that the elastic state due to interactions across twin boundaries does not differ from that across conventional grain boundaries. The component of the Type II stress in twins or grains that is normal to the boundary exhibits very large fluctuations and appears uncorrelated with the crystallographically induced elastic anisotropy. Twin boundaries were specifically selected for this study because the boundary normal is the elastically stiffest direction in both parts of the twin. In addition twinned grains often have a lamellar structure, meaning that one part of a twin is connected to the other across two boundaries, as also seen in Figure 1(b). The absence of special correlations even in this case therefore implies that elastic grain interactions are complex, and that the elastic-regime stress state of a grain is a function of the entire grain neighbourhood.

The investigated twins are coarse and differ in scale from nanotwinned metals. Young's modulus of nanotwinned samples has been found in simulations to be size-dependent [19], indicating that twins in some cases may affect even the macroscopic Type I stress of the sample.

The annealing twins studied also differ fundamentally from deformation-induced twins, which are generated as a plastic response to the applied stress. For deformation twins it has been shown that the Type II stress state of a grain provides a better estimate than Type I stresses of the twin variant selection $[20,21]$. However, localised transmission of slip and twinning across grain boundaries also plays a role $[22,23]$. Local stress concentrations at the micron and submicron scales induced by slip bands have indeed been observed at grain boundaries using high-resolution techniques [24,25]. With respect to the Type II stress states of parent and deformation-induced twins, these have been shown to differ significantly $[26,27]$, whereas equilibrium of the more local Type III stresses across deformation-induced twin boundaries have been observed with a sub-micrometre spatial resolution [28]. The spatial resolution of the current approach does not enable an experimental investigation of Type III stresses at the boundaries of annealing twins.

In conclusion, the grain-resolved Type II elastic-regime stresses in individual grains have been analysed for interactions across twin and grain boundaries. The boundaries connect compliant and stiff parts and have a common macroscopic inclination to the tensile axis. The stress components normal to the boundary plane in the two twin parts or grains are uncorrelated in both cases, demonstrating that a single neighbour has a limited influence on the Type II stress. Elastic grain interactions are, however, strong as the stress states of neighbouring grains were found to exhibit very large variations. These must arise from complex interactions with the entire set of neighbouring grains. The tensile stress component also demonstrates a large spread, but a qualitative effect of the crystallographic direction of the tensile axis is observed. This trend corresponds to elastic interaction in between the limits of Voigt and Reuss. The results also imply that twin and grain boundaries may be treated in the same manner in future polycrystal elastic-regime modelling efforts, in spite of the common elastically stiff direction shared across a twin boundary. This is of particular importance to modelling at scales not considering spatially localised phenomena, e.g. self-consistent approaches. 


\section{Figure captions}

Figure 1 Full 3D grain map of (a) the illuminated $0.7 \times 0.7 \times 0.5 \mathrm{~mm}^{3}$ section of the sample, (b) a selected twin pair (enclosing box $0.15 \times 0.15 \times 0.15 \mathrm{~mm}^{3}$ ) and (c) a representative pair of neighbouring grains (enclosing box $0.15 \times 0.15 \times 0.15 \mathrm{~mm}^{3}$ ) colour coded according to the orientation of the tensile axis. The tensile stress is applied along the $z$-direction.

Figure 2 Stereographic triangles marking the crystallographic direction of the tensile axis of the grains mapped. Colours refer to the grain average values of tensile a) elastic strain and b) stress. Volume weighted averages are $0.096 \%$ and $174 \mathrm{MPa}$, which agree well with the macroscopically measured values of $0.12 \%$ and $168 \mathrm{MPa}$.

Figure 3 Orientation of the tensile axis in the selected (a) twin pairs and (b) pairs of neighbouring grains. In (a) the red diamonds represent the compliant part of the twin pair that relates to a stiffer part represented by a blue dot for $1.7 \leq \mathrm{E}_{\text {stiff }} / \mathrm{E}_{\text {compl }}$ or a green square for $1.2<E_{s t i f f} / E_{c o m p l}<1.7$. In (b) each grain represented by a red diamonds neighbours a grain represented by a grey hexagon.

Figure 4 Stress normal to (a) twin planes in selected twin pairs (cf. Figure 1(b)) and (b) selected grain boundary planes (cf. Figure $1(c))$. The $x$-axis shows the stress in the compliant part (red in Figure 3 ) while the $y$-axis gives the stress in the corresponding stiffer twin or grain for (a) or (b) respectively.

\section{Acknowledgements}

NYJ, GW and JO acknowledge support from the Danish Independent Research Council - Technology and Productions Sciences grant n. DFF-13555-00220. The Danish Agency for Science, Technology and Innovation is acknowledged for covering the expenses in relation to the synchrotron experiment (through Danscatt). PS acknowledges support from the Materials \& Manufacturing Directorate of the U.S. Air Force Research Laboratory. This work is based upon research conducted at the Cornell High Energy Synchrotron Source (CHESS), which is supported by the NSF \& NIH/NIGMS via NSF award DMR-1332208.

\section{References}

[1] H.F. Poulsen, S.F. Nielsen, E.M. Lauridsen, S. Schmidt, R.M. Suter, J. Appl. Crystallogr. 34 (2001) 751.

[2] H.O. Sørensen, S. Schmidt, J.P. Wright, G.B.M. Vaughan, S. Techert, E.F. Garman, J. Oddershede, J. Davaasambu, K.S. Paithankar, C. Gundlach, H.F. Poulsen, Zeitschrift Fur Krist. 227 (2012) 63.

[3] J.C. Schuren, P.A. Shade, J. V Bernier, S.F. Li, B. Blank, J. Lind, P. Kenesei, U. Lienert, R.M. Suter, T.J. Turner, D.M. Dimiduk, J. Almer, Curr. Opin. SOLID STATE Mater. Sci. 19 (2015) 235.

[4] S. Schmidt, U.L. Olsen, H.F. Poulsen, H.O. Sørensen, E.M. Lauridsen, L. Margulies, C. Maurice, D. Juul Jensen, Scr. Mater. 59 (2008) 491.

[5] R. Pokharel, J. Lind, S.F. Li, P. Kenesei, R.A. Lebensohn, R.M. Suter, A.D. Rollett, Int. J. Plast. 67 (2015) 217.

[6] J. Oddershede, S. Schmidt, H.F. Poulsen, H.O. Sørensen, J. Wright, W. Reimers, J. Appl. Crystallogr. 43 (2010) 539.

[7] P. Hedström, T.-S. Han, U. Lienert, J. Almer, M. Odén, Acta Mater. 58 (2010) 734.

[8] J. Oddershede, S. Schmidt, H.F. Poulsen, L. Margulies, J. Wright, M. Moscicki, W. Reimers, G. Winther, Mater. Charact. 62 (2011) 651. 
[9] J. V. Bernier, N.R. Barton, U. Lienert, M.P. Miller, J. Strain Anal. Eng. Des. 46 (2011) 527.

[10] M.K.A. Koker, U. Welzel, E.J. Mittemeijer, Philos. Mag. 93 (2013) 2967.

[11] M.K.A. Koker, U. Welzel, E.J. Mittemeijer, J. Appl. Crystallogr. 47 (2014) 391.

[12] P.A. Shade, B. Blank, J.C. Schuren, T.J. Turner, P. Kenesei, K. Goetze, R.M. Suter, J. V Bernier, S.F. Li, J. Lind, U. Lienert, J. Almer, P.A. Shade, B. Blank, J.C. Schuren, T.J. Turner, P. Kenesei, K. Goetze, R.M. Suter, J. V Bernier, S.F. Li, J. Lind, U. Lienert, J. Almer, Rev. Sci. Instrum. 86 (2015) 093902.

[13] J.H. Lee, C.C. Aydiner, J.D. Almer, J.V. Bernier, K.W. Chapman, P.J. Chupas, D.R. Haeffner, K. Kump, P.L. Lee, U. Lienert, A. Miceli, G. Vera, J. Synchrotron Radiat. 15 (2008) 477.

[14] M. Guizar-Sicairos, S.T. Thurman, J.R. Fienup, Opt. Lett. 33 (2008) 156.

[15] S. Schmidt, J. Appl. Crystallogr. 47 (2014) 276.

[16] H.M. Ledbetter, Br. J. Non-Destructive Test. 23 (1981) 286.

[17] W. Voigt, Lehrbuch Der Kristallphysik, Teubner, Leipzig, 1910.

[18] A. Reuss, Zeitschrift Für Angew. Math. Und Mech. 9 (1929) 49.

[19] D. Shen, J. Du, R. Melnik, B. Wen, Comput. Mater. Sci. 89 (2014) 24.

[20] H. Abdolvand, M. Majkut, J. Oddershede, J. Wright, M.R. Daymond, Acta Mater. 93 (2015) 235.

[21] H. Abdolvand, M. Majkut, J. Oddershede, J. Wright, M.R. Daymond, Acta Mater. 93 (2015) 246.

[22] T.R. Bieler, L. Wang, A.J. Beaudoin, P. Kenesei, U. Lienert, Metall. Mater. Trans. A Phys. Metall. Mater. Sci. 45 (2014) 109.

[23] A. Khosravani, D.T. Fullwood, B.L. Adams, T.M. Rampton, M.P. Miles, R.K. Mishra, Acta Mater. 100 (2015) 202.

[24] T. Benjamin Britton, A.J. Wilkinson, Acta Mater. 60 (2012) 5773.

[25] Y. Guo, D.M. Collins, E. Tarleton, F. Hofmann, J. Tischler, W. Liu, R. Xu, A.J. Wilkinson, T.B. Britton, Acta Mater. 96 (2015) 229.

[26] C.C. Aydıner, J. V. Bernier, B. Clausen, U. Lienert, C.N. Tomé, D.W. Brown, Phys. Rev. B 80 (2009) 024113.

[27] L. Wang, J. Lind, H. Phukan, P. Kenesei, J.-S. Park, R.M. Suter, A.J. Beaudoin, T.R. Bieler, Scr. Mater. 92 (2014) 35.

[28] L. Balogh, S.R. Niezgoda, A.K. Kanjarla, D.W. Brown, B. Clausen, W. Liu, C.N. Tomé, Acta Mater. 61 (2013) 3612. 


\section{Graphical Abstract}
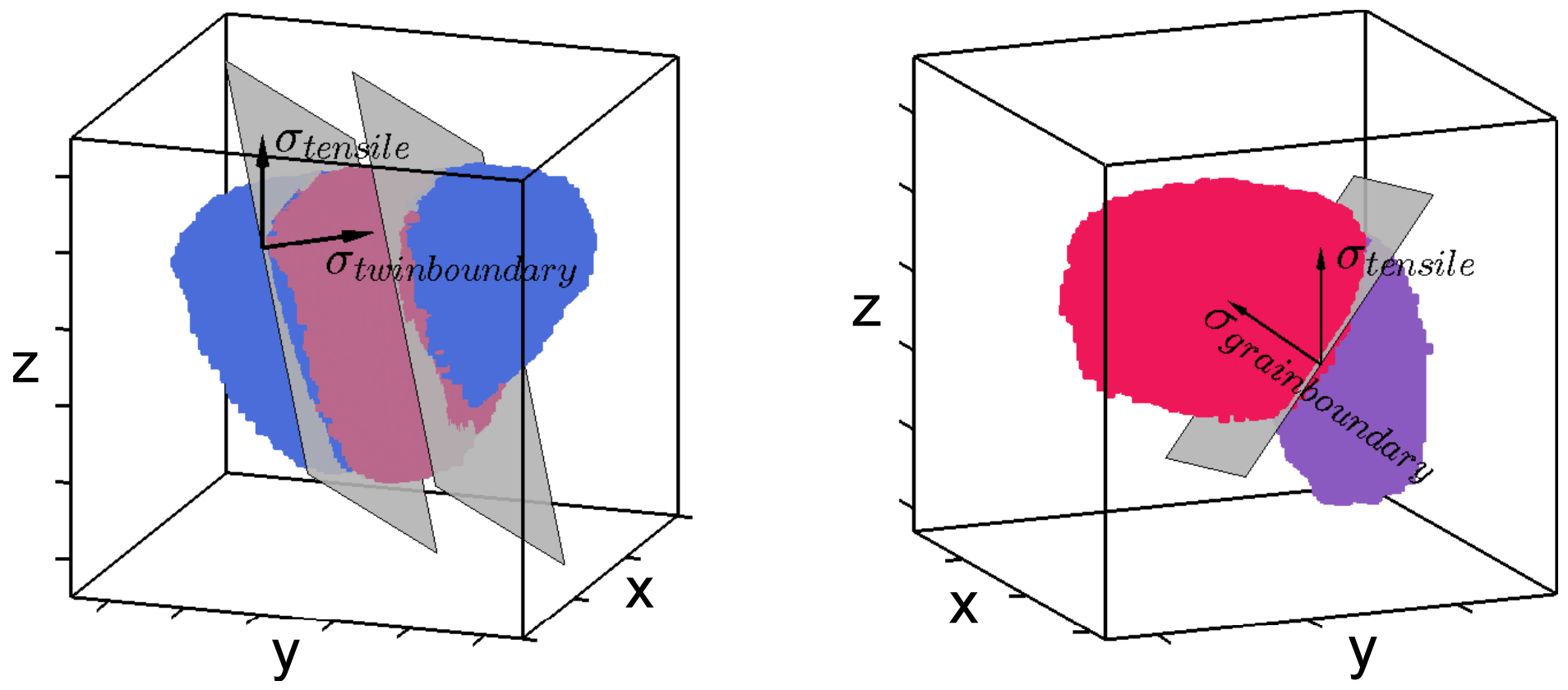
(a)

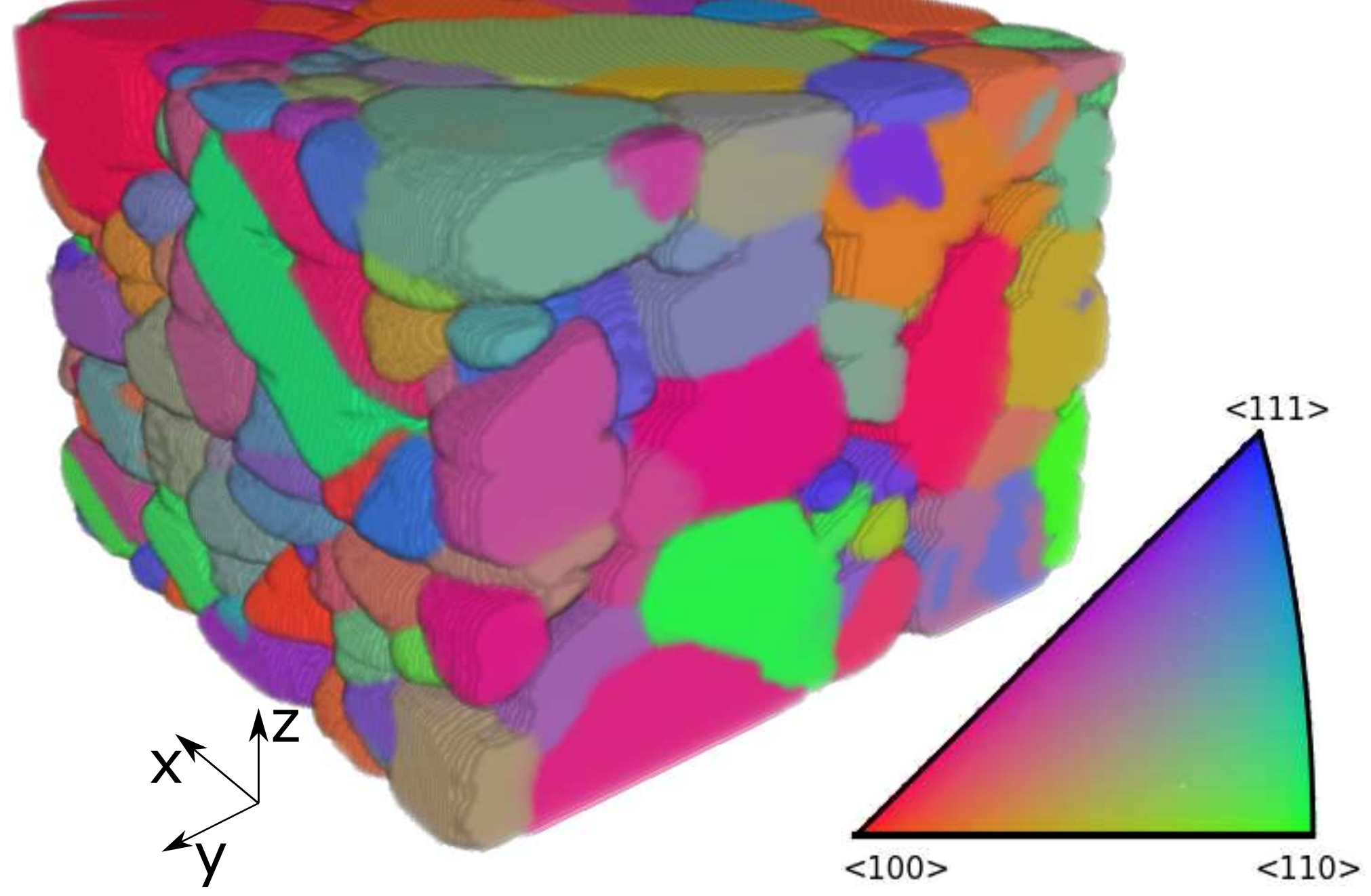

(b)

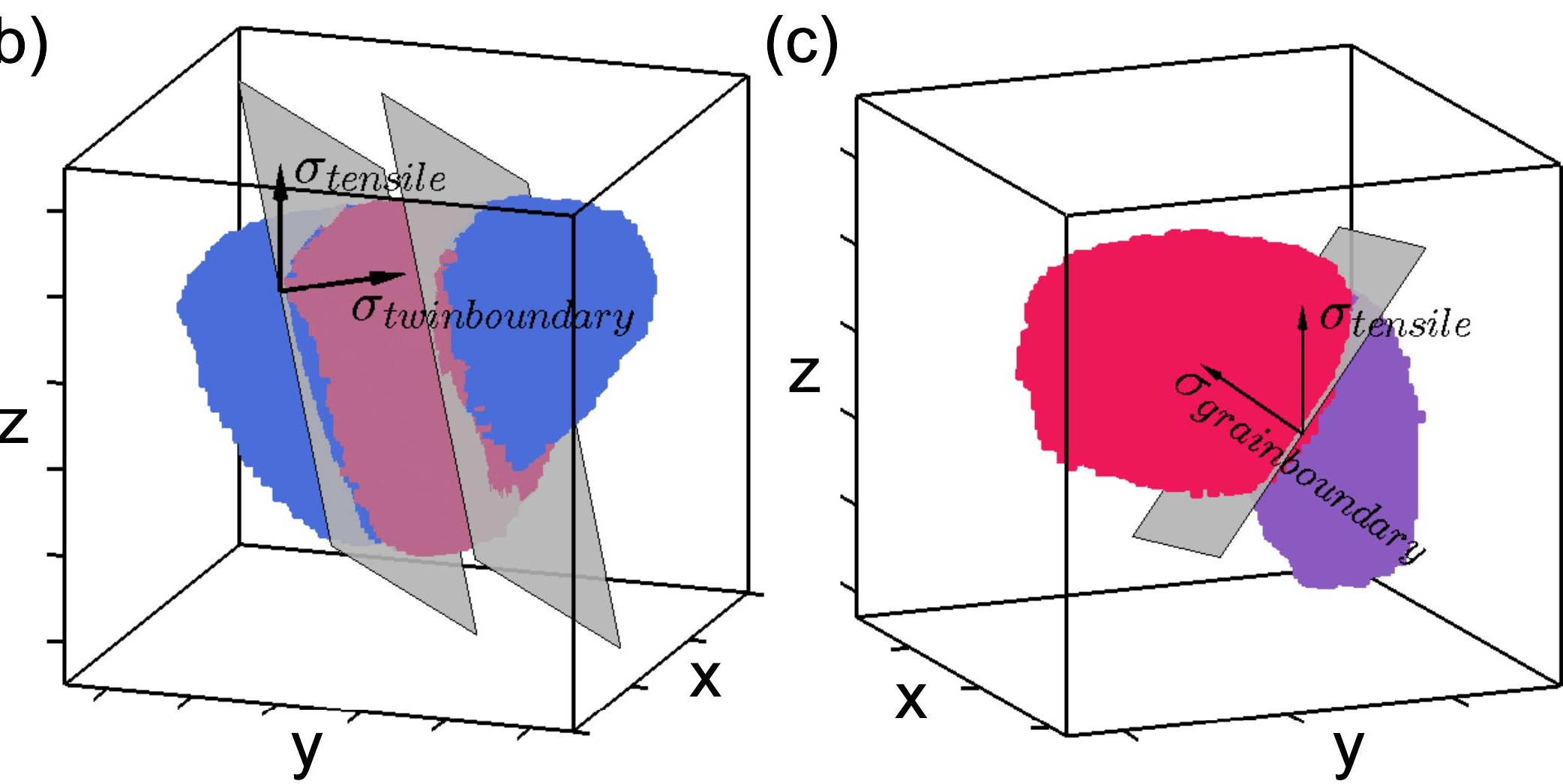




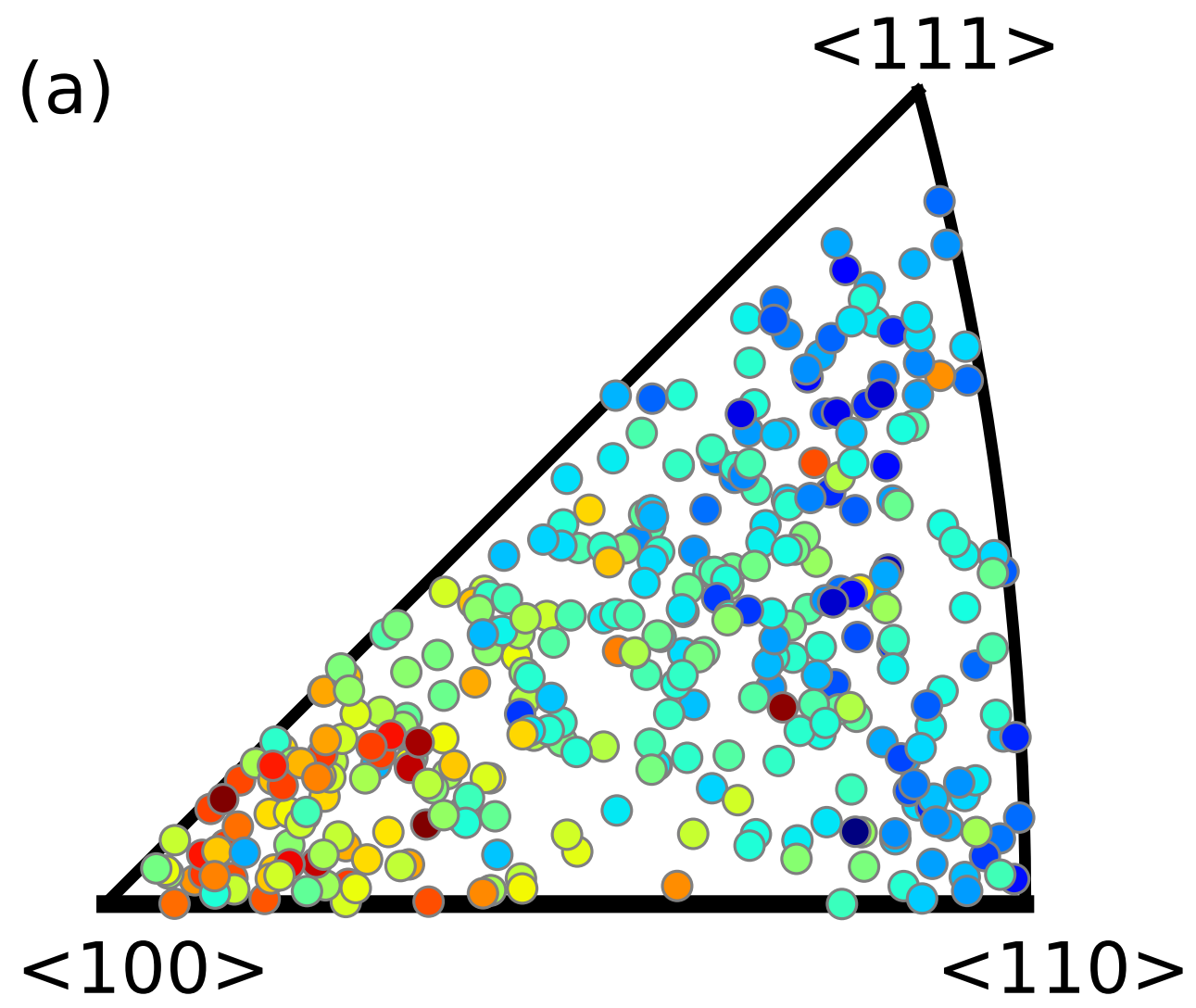

$\epsilon$ [\%]
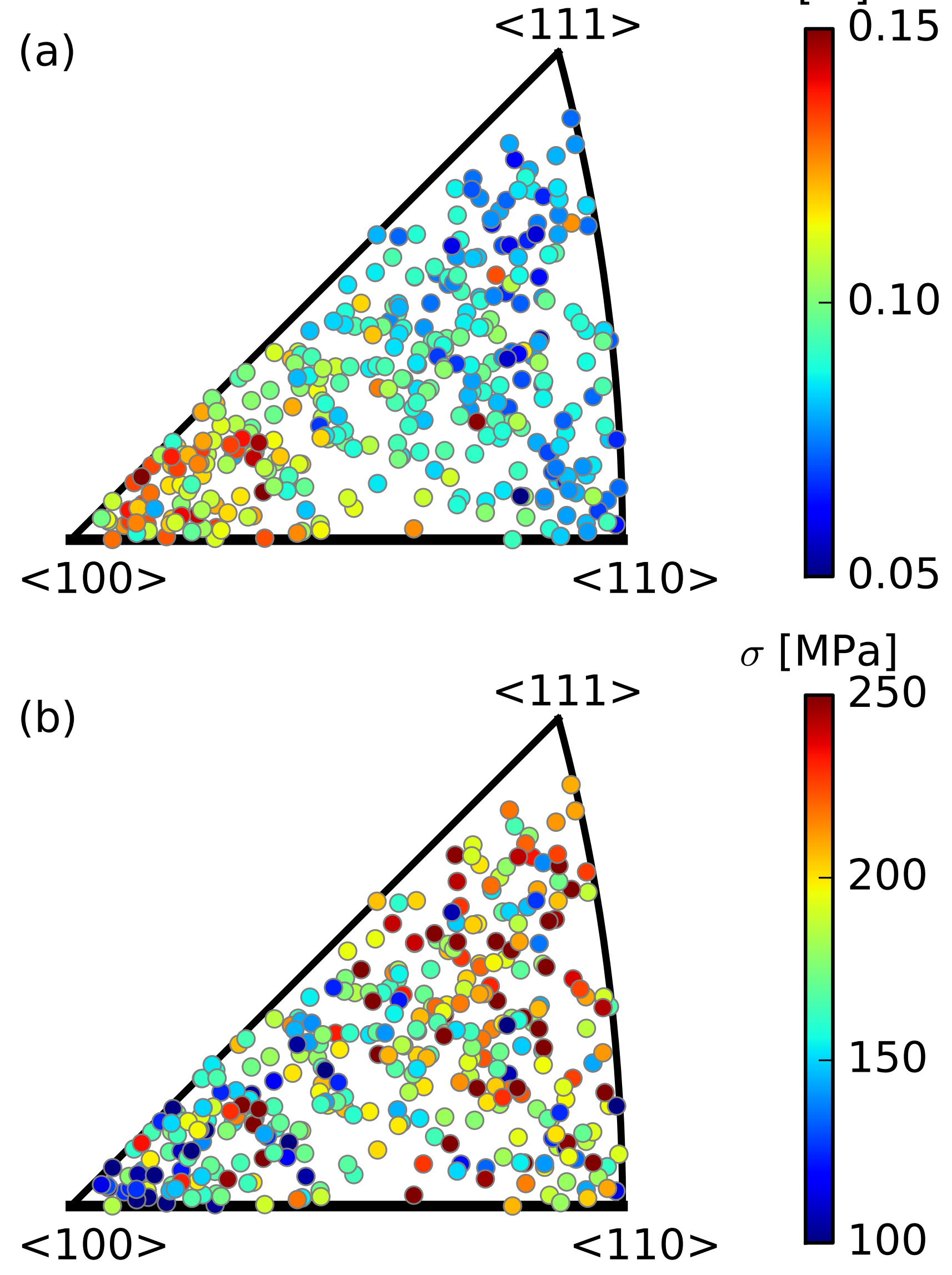


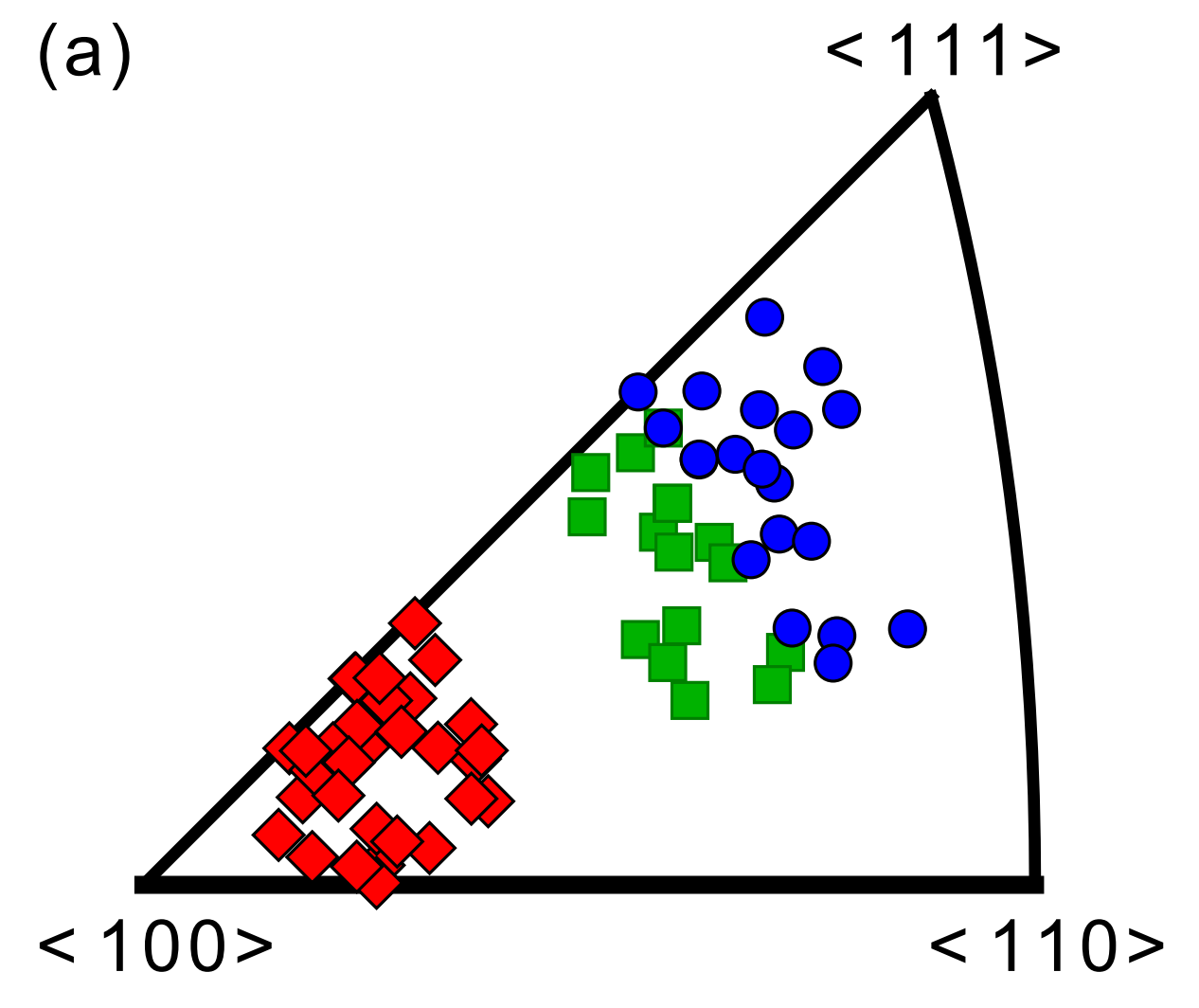

(b)

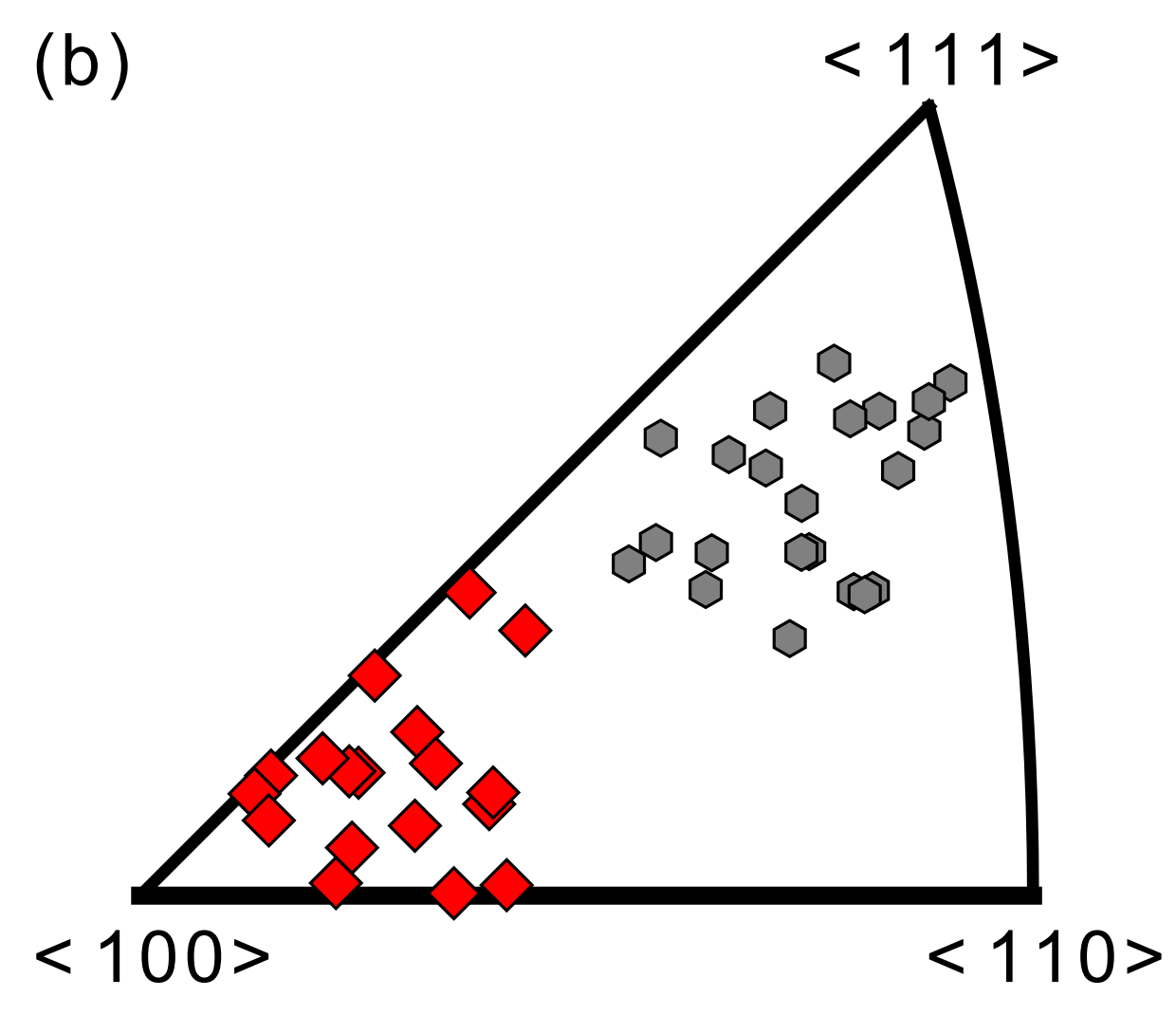

$<100>$

$<110>$

Figure 3

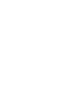



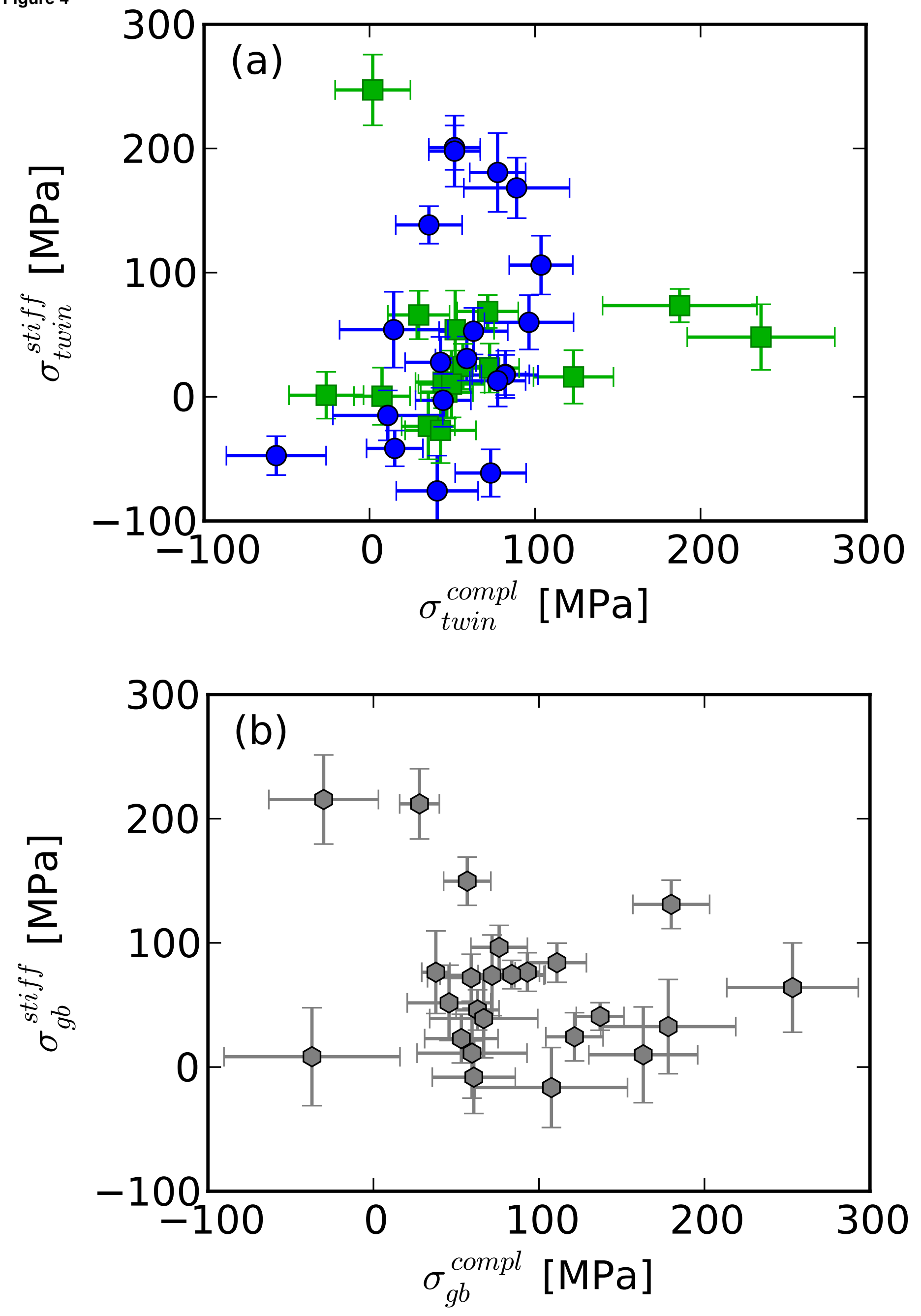